\title{
BMJ Open Rapid ethnographic assessment of the COVID-19 pandemic April 2020 'surge' and its impact on service delivery in an Acute Care Medical Emergency Department and Trauma Center
}

To cite: Palinkas LA,

Whiteside L, Nehra D, et al. Rapid ethnographic assessment of the COVID-19 pandemic April 2020 'surge' and its impact on service delivery in an Acute Care Medical Emergency Department and Trauma Center. BMJ Open 2020;10:e041772. doi:10.1136/ bmjopen-2020-041772

- Prepublication history and additional material for this paper are available online. To view these files, please visit the journal online (http://dx.doi org/10.1136/bmjopen-2020041772).

Received 16 June 2020 Revised 23 September 2020 Accepted 05 October 2020

Check for updates

(C) Author(s) (or their employer(s)) 2020. Re-use permitted under CC BY-NC. No commercial re-use. See rights and permissions. Published by BMJ.

For numbered affiliations see end of article.

Correspondence to Dr Lawrence A Palinkas; palinkas@usc.edu

\section{ABSTRACT}

Objectives Assess the impacts of the COVID-19 pandemic on service delivery by frontline healthcare providers in acute care medical and emergency department settings and identify strategies used to cope with pandemic-related physical and mental health demands.

Design Rapid clinical ethnography of patient-provider encounters during an initial pandemic 'surge' conducted by a team of clinician-researchers using a structured protocol for qualitative data collection and analysis. Setting Level 1 trauma centre at Harborview Hospital in Seattle Washington in April 2020.

Participants Frontline clinical providers serving as participant observers during performance of their clinical duties recorded observations and summaries of conversations with other providers and patients.

Results We identified four different kinds of impacts: procedural, provider, patient and overall. Each impact highlighted two or more levels of a socioecological model of services delivery: (1) the epidemiology of COVID-19, (2) outer setting, (3) inner or organisational setting and (4) individual patient and provider. Despite significant changes in procedures that included COVID-19 screening of all admitted patients, social distancing and use of personal protective equipment, as well as changes in patient and provider behaviour, the overall impact of the pandemic on the emergency department and acute care service delivery was minimal. This is attributed to having a smaller surge than expected, a quick response by the healthcare system to anticipated demands for service delivery and protection of patients and providers, adequate supplies and high provider morale.

Conclusions Although limited to one setting in one healthcare system in one community, the findings offer some important lessons for healthcare systems that have yet to be impacted as well as systems that have been more severely impacted. Each of the socioecological framework levels was found to impact service delivery to patients, and variations at each of these levels account for variations in that quality of care globally.

\section{Strengths and limitations of this study}

- We conducted a rapid clinical ethnography of patient-provider encounters during an initial COVID-19 pandemic 'surge' in Seattle Washington to assess the impacts on service delivery by frontline healthcare providers in acute care medical and emergency department settings and identify strategies used to cope with pandemic-related physical and mental health demands.

- The COVID-19 outbreak resulted in significant changes in acute care clinical procedures, the behaviours of patients and providers and overall healthcare system performance that was influenced by four different levels of a socioecological model of service delivery at a healthcare system that was one of the first in the USA to be impacted by the pandemic.

- Providers reported widespread anxiety related to infection and transmission of COVID-19 to family members, along with depression related to perceived limitations to delivering care and stress related to the pandemic's financial impacts and prolonged isolation and confinement.

- Providers also reported widespread use of coping strategies and resources to prevent disease spread and deliver high-quality healthcare.

- Although limited to one setting in a single US healthcare system where the impacts associated with the pandemic have not been as severe to date as has been the case elsewhere, the findings also offer important lessons for healthcare system providers responding to the COVID-19 pandemic in other settings across the globe.

\section{INTRODUCTION}

In January 2020, the WHO announced the emergence of a novel COVID-19 in Wuhan, China. ${ }^{1}$ Since then, COVID-19 has become a global pandemic on a scale not seen since the 1918 influenza pandemic, which led to an estimated 50000000 deaths. ${ }^{2}$ As of 28 August 
2020, there were over 24.5 million confirmed cases of COVID-19 and 832748 deaths across the globe; the USA is perhaps the most severely impacted nation with more than 5.8 million confirmed cases and 181022 deaths. ${ }^{3}$ In most states, all non-essential businesses and services were closed and employees were laid off or furloughed, resulting in a national unemployment rate of $14.7 \%$ in April 2020. ${ }^{4}$ Social distancing and use of face masks, closure of non-essential businesses and mandated quarantines and sheltering in place have been used to control the spread of the disease ${ }^{5}$

Along with other forms of natural disasters and acts of terrorism, infectious disease outbreaks or pandemics often result in a surge in demand for medical care, beginning with emergency departments (EDs). ${ }^{6}$ Healthcare systems generally plan responses to such surges by having a pandemic preparedness plan in place for triaging and caring for exposed patients. However, studies that have examined the impact of infectious disease outbreaks on service delivery have generally been retrospective and focused on patterns of admissions and discharges in EDs. ${ }^{6-8}$ To date, there have been no studies conducted during a pandemic that have focused on the challenges to delivering acute care services and the extent to which these challenges were addressed by system policies and individual provider practices.

One of the potential influences of infectious disease outbreaks on service delivery in acute care settings is diminished performance due to stress and decrements in mental health. Burnout in healthcare professionals is frequently associated with poor-quality care. ${ }^{9}{ }^{10}$ Frontline healthcare providers currently responding to the exponential increase in demands for care associated with the COVID-19 pandemic share many of the same risk factors for adverse mental health outcomes as those responding to other forms of disaster. ${ }^{61112}$ Several studies of infectious disease outbreaks, including the 2003 SARS outbreaks in Asia and Canada and the 2012 Middle East Respiratory Syndrome (MERS) outbreak in Saudi Arabia, have documented elevated levels of stress, anxiety, depression and post-traumatic stress disorder, ${ }^{13-19}$ which often persist years after the outbreak. ${ }^{20}{ }^{21}$ Lack of social support and communication, maladaptive coping and lack of training were important risk factors for developing negative psychological outcomes across all types of disasters.

However, the current COVID-19 pandemic is unique in several respects. The number of cases testing positive for the novel coronavirus and the number of hospital admissions and deaths have exceeded that of previous respiratory disease pandemics, including SARS and MERS, and differ from these pandemics in terms of infectious period, transmissibility, clinical severity and extent of community spread..$^{22}$ In an effort to 'flatten the curve' of disease transmission, morbidity and mortality, healthcare providers will be exposed for a longer period of time than is the case in other pandemics ${ }^{23}$ Frontline providers are confronting the possibility of becoming infected themselves, thereby increasing the risk of coronavirus-related morbidity and mortality, and preventive measures such as social distancing will likely impact both personal and professional behaviours. A recently published investigation of mental health outcomes among healthcare workers in Wuhan, China found that engagement in direct diagnosis, treatment and care of patients with COVID-19 were associated with a higher risk of symptoms of depression, anxiety, insomnia and distress. ${ }^{24}$ Although these features of the current pandemic have been prominent in the news media, ${ }^{25}$ to date, there have been no systematic studies of these impacts on service delivery. Moreover, the focus of media attention has been on healthcare systems in locations like New York City and in Spain and Italy that have been most severely impacted by the number of patients testing positive for COVID-19. Little is known of its impacts on healthcare systems in communities where the outbreak has been less dramatic to date and how frontline providers in these systems are coping with these impacts.

To address the lack of information on these issues, we used a novel technique for conducting a rapid ethnographic assessment of the impacts of the COVID-19 pandemic on physicians and staff of a level 1 trauma centre of Harborview Medical Center in Seattle Washington that was among the first in the USA to be impacted by the pandemic. ${ }^{26}$ Our study had two aims: (1) assess the impacts of the COVID-19 pandemic on service delivery by frontline healthcare providers working in acute care medical and ED settings at the trauma centre and (2) identify strategies being used by these providers to cope with the increased physical and mental health demands associated with the pandemic. Our examination of impacts and strategies was guided by a conceptual framework grounded in the social-ecological model of behaviour. This model argues that individual behaviour is shaped by factors at multiple levels, including institutional, community and policy levels in addition to intrapersonal and interpersonal levels. ${ }^{27}$ In this instance, the individual behaviour is that of the providers and patients who define the quality of care provided by one individual (the healthcare provider) and received by another individual (the patient). ${ }^{28}$ The social-ecological model has been also used in other studies of health services delivery in ED settings. ${ }^{29}$

\section{METHODS \\ Design overview}

The investigation reported here was embedded within a larger randomised comparative effectiveness trial of the impact of a peer-integrated acute care to primary care and community care coordination intervention. ${ }^{30}$ To assess implementation of the evidence-based interventions, we used a mixed methods protocol that incorporates principles of rapid assessment procedures and clinical ethnography. ${ }^{31}$ The Rapid Assessment Procedure Informed Clinical Ethnography (RAPICE) approach was previously used to describe primary and secondary 
COVID-19 preventive interventions as well as ethical tensions and stepped coping strategies in the early days and weeks of the pandemic. ${ }^{32}$ In the study reported here, RAPICE was used because the research team had already been trained in its use and had collected ethnographic data at the trauma centre related to the parent study prior to the COVID-19 outbreak, ${ }^{31}$ it was originally developed as a tool to iteratively assess and inform care delivery during mass violence events ${ }^{33}$ and natural disasters, ${ }^{34}$ it could be implemented with minimal additional resources within the framework of the larger comparative effectiveness trial, it is a minimally invasive form of data collection that can be used when priority was given to service delivery, and it can provide a depth of understanding to the challenges faced in service delivery not available from quantitative surveys.

\section{Participants}

Study participants were patients and providers who interacted with or otherwise were observed by members of the parent study research team $(n=5)$ engaged in the delivery of care within the trauma centre at Harborview Medical Center during a COVID-19-related April 2020 'surge'. The facility is the only designated level I trauma and burn centre in Washington state and is the regional trauma and burn referral centre for Alaska, Montana and Idaho. The 412-bed facility has around 17000 admissions, 259000 clinic visits and $59000 \mathrm{ED}$ visits annually. ${ }^{35}$ During the month of April 2020, the hospital had 1089 total admissions. On average, the daily COVID-19 census was 18 patients (range $=10-26$ patients). Research team members included a trauma surgeon, ED physician, trauma centre nurse manager, acute care medical consultation-liaison psychiatrist and social worker, each of whom served as participant observers (POs) in the trauma centre. Each team member had an opportunity to observe various components of acute care delivery, from triage management and emergency care to surgical procedures, in-hospital mental health service delivery and trauma centre to primary care linkages. Participants were given training by the first author to assume the role of POs during their shifts in the trauma centre. This training included the principles and practice of RAPICE, what information to collect and how (ie, through observation and informal interviews with other providers and staff), how to record information collected in field jottings and field notes and how to acknowledge and manage the researcher's subjectivity through reflexivity or systematic awareness of the potential for bias and distortion. ${ }^{36}$

\section{Data collection}

Data included observations and interactions with patients and other providers made while engaged in delivering routine clinical services. POs were charged with observing and recording events that illustrate the impacts of the pandemic on provider performance and well-being and on provider interactions with patients, family members and other providers. They also informally collected reports from other acute care providers and staff of physical and emotional impacts of additional workload. Finally, POs were asked to obtain information on strategies used by providers to cope with the increased personal and professional demands imposed by the pandemic. The trauma centre providers and staff were aware of the PO's role as researchers involved in the parent study and the focus of their investigation per approval by the Institutional Review Boards of the University of Washington and University of Southern California (UP-20-00298) prior to the initiation of the investigation. Informed consent from the POs themselves was obtained from the first author.

Information on these observations and interactions were recorded through periodic jottings summarising observations and interactions and more detailed field notes that could be updated each day. Field notes also included impressions of events observed and exchanges with other providers and staff as well as preliminary interpretations of the significance of these events and exchanges. Each PO then participated in a semistructured debriefing interview with the first author to clarify and expand on information contained in jottings and field notes and provide a preliminary interpretation of their observations and interactions. A copy of the debriefing interview guide is provided as in online supplemental document 1 . Debriefs lasting between 50 and $60 \mathrm{~min}$ in duration were conducted using the Zoom video conferencing platform, recorded and transcribed for analysis. Written copies of debriefs were then provided to the POs, enabling them to revise or elaborate on comments made.

\section{Data analysis}

The first author reviewed all data collected by the POs and performed a preliminary analysis, using the immersions/crystallisation, ${ }^{37}$ and focused thematic analysis techniques $^{38}$ that are part of the RAPICE methodology. ${ }^{31}$ The first author reviewed the data and then queried each PO during the debrief to gain more insight into the data and its context and to obtain a preliminary interpretation of the meaning and significance of data provided by the PO. Two hundred and sixty-eight double-spaced pages of field notes, jottings, memos, documents and transcripts of the member-checking debriefing interviews collected over a 4 week period were then coded by the first author to condense the data into analysable units. Segments of text ranging from a phrase to several paragraphs were assigned codes based on a priori (eg, from a semistructured interview guide) or emergent themes (also known as open coding). Following the open coding, codes were assigned to describe connections between and within categories (also known as axial coding). Based on these codes, QSR NVivo V.12 was used to generate a series of themes arranged in a treelike structure connecting text segments grouped into separate categories of codes or 'nodes'. Consistent with previously explicated RAPICE methods, ${ }^{31}$ a discussion then ensued until both the POs and the first author reached consensus as to the meaning and significance of the data. 


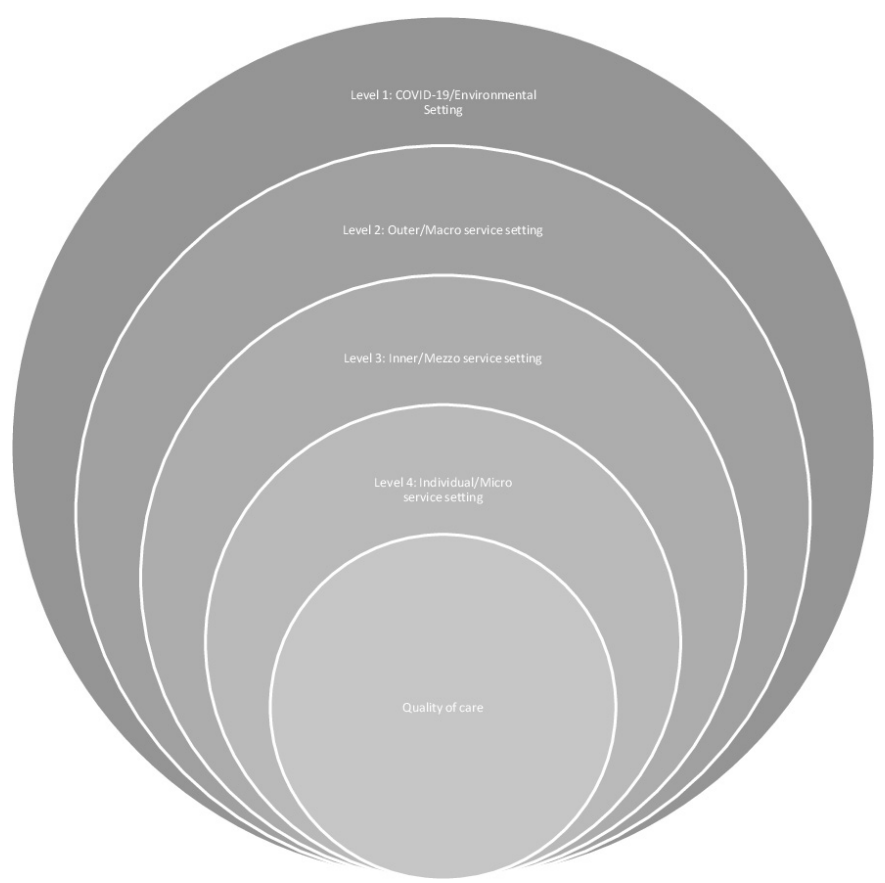

Figure 1 Conceptual framework of COVID-19 pandemic and its impact on supply and demand for mental and behavioural health services.

\section{Patient and public involvement}

Patients and the public were not involved in the design or execution of this study.

\section{RESULTS}

Overall, our analysis revealed four broad impacts of the COVID-19 pandemic on service delivery: (1) impacts on procedures, (2) impacts on providers, (3) impacts on patients and (4) overall impacts on quality of care. Each of these themes is linked together at four broad levels of a socioecological model of influences on patient care, illustrated in figure 1.

The outermost or environmental level is dictated by the novel coronavirus and its global spread and includes the nature of virus transmission; social and biological characteristics of risk and resilience; public health guidelines for preventing the spread of infection; risk of reinfection; disease sequalae; survival rates and clinical outcomes. The second level is the external or macroservice setting that has dictated the supply (eg, availability of personnel and equipment like personal protective equipment (PPE) and ventilators) and demand (eg, number of patients seen overall, patients who test positive for COVID-19 or are under investigation for having COVID-19 and the nature of the problems seen). The third level is the internal or mezzo service setting of the healthcare system and includes the availability of beds to handle increased demand, healthcare system guidelines and policies put in place to ensure the safety and health of both patients and providers and the transition to delivery of services using telehealth platforms to reduce the need for patients to be physically present at the hospital. The fourth level is that of the individual provider and patient or microservice setting and includes variations in the demands placed on individuals that include the anxiety related to fear of infection, depression, ethical conflicts, social tension and stress and the resources and strategies used by individuals to cope with these demands.

\section{Theme 1: impacts on procedures}

The first theme of impacts on procedures and quality of care can be divided into three subthemes: (1) challenges related to testing patients for COVID-19, (2) altering procedures to insure adequate social distancing and (3) use of PPE. Each of these represents the interconnections between levels 1-4 described above and are examined in detail below. Illustrative quotations from fieldnotes and interviews for each subtheme are provided in table 1.

\section{COVID-19 testing}

The implementation of a policy that all patients requiring acute care undergo testing for COVID-19 because of a need to preserve PPE for confirmed patients with COVID-19 or patients at high risk for COVID-19 has resulted in delays in getting treatment for often lifethreatening conditions. For patients with severe mental health issues, getting consent to perform testing has been problematic. Especially challenging for providers has been patients showing symptoms that are similar to those of COVID-19, such as withdrawal from heroin or other illicit substances. Although the delays in getting treatment do not appear to have compromised the quality of care received, providers expressed concern that patients needing urgent but not immediate attention become sicker while awaiting COVID-19 test results. Experience with guideline implementation and its effects on workflow and service delivery, along with information from other healthcare systems, led to changes in guidelines and protocols for COVID-19 screening. Changes in guidelines resulted in delays in delivering care and confusion over what guidelines were in effect at any point in time.

\section{Social distancing}

According to the centres for disease control, social distancing, also called 'physical distancing', means keeping a safe space between yourself and other people who are not from your household. ${ }^{39}$ To practice social or physical distancing, the Centers for Disease Control and Prevention (CDC) recommends that one stay at least six feet (about two arms' length) from other people who are not from your household in both indoor and outdoor spaces. Within the trauma centre, social distancing included protocols and procedures designed to minimise person-to-person contact.

Imposition of social distancing guidelines for the benefit of both patients and providers led to several changes in procedures, including reducing the need for patients to come to ED and suspension of non-essential procedures. Social distancing guidelines also impacted patterns of interactions among providers. Routine interactions such 
Table 1 Impacts of COVID-19 pandemic on clinical procedures

\begin{tabular}{lll}
\hline Subtheme & Level & Illustrative quote \\
\hline $\begin{array}{l}\text { Covid testing } \\
\text { Delay in care }\end{array}$ & 1,3 & $\begin{array}{l}\text { Any trauma who is intubated (which is most of our sick trauma patients) is considered } \\
\text { COVID positive coming in and we have to perform the initial resuscitation and evaluation } \\
\text { in airborne precautions and limit people/supplies in the room. This can sometimes cause a } \\
\text { delay in some of the care. - fieldnote }\end{array}$
\end{tabular}

Impact on quality of $1,2,3$ care
... sometimes patients have you know what normally we would consider to be relatively urgent things and we would just get the patient down to the OR quickly because there is the potential for them to decompensate. They might not be dying in front of you, but there is the potential for them to decompensate. And that sort of decision of like 'hey should we like in this situation to preserve PPE, like get this COVID test and wait because we think the patient's kind of going to be able to make it a few hours without decompensating,' that I find kind of challenging because it feels like you're sometimes providing maybe not the best care because normally you would go straight down to the operating room but there's also all these layers of if I do that, you know it uses this much more PPE and what not.- debriefing interview

Guideline uncertainty $1,3,4$ Constantly evolving pathways for COVID testing and clearance which is understandable but no clear consensus on a day to day basis, or at least a lot of confusion. - fieldnote

\section{Social distancing}

Impact on

procedures

I think, you know, we're a teaching hospital so anything that happens, anything that happened, I should say in the past, happened with a large group of people. You know there's the people who are performing the task and then the observers who are learning. The observers are no longer present for any of that. And even the activities that are being provided have been rethought to a point where we can pare them down to just the minimum number required. And so, so yes absolutely. There's a significant amount of workflow changes that occurred to minimize the numbers of people that are involved.debriefing interview

$\begin{array}{lll}\begin{array}{l}\text { Reducing patient } \\ \text { need to visit ED }\end{array} & 1,2,3 & \begin{array}{l}\text { Worked with patient to avoid ER a few weeks ago after a fall by coordinating nurse \& } \\ \text { doctor phone call; resulted in patient creating sling and icing injury. Resolved without visit } \\ \text { to ER. Pt needs to go to doctor \& physical therapy often for pain management and routine } \\ \text { care for chronic conditions. Clinics do not want her coming in because not "absolutely } \\ \text { necessary."-jotting }\end{array}\end{array}$

Impacts on provider $1,3,4$ interactions

Also, we note the geography of our ED has changed so keep $>6$ feet of space between patients and allow for providers in patient care areas, so providers no longer congregate together in non-clinical spaces and sit separate from nurses which decreases clinical communication. There were no bad outcomes, just notable how much harder it is to communicate as a whole clinical team. - fieldnote

Reduced presence of $1,3,4$ family members

And then I really think one of the biggest things that's been sort of hard I think for us as a group and I think for all healthcare providers sort of who are taking care of any patient, COVID positive or not, is that, is the fact that you know we really aren't able to have family members in the hospital almost at all, which is a very different way than we usually practice. And that's been really hard I think on everyone in sort of the hospital but also the patients and their families. - debriefing interview

Use of telehealth $\quad 1,3,4 \quad$ Before, when all this started we were not set up for telehealth in anyway, we did do phone calls that's always been something but it was seen as only, we only did that if there was some really extenuating circumstances, or if something was so minor that it just seemed better to do it over phone. So as soon as really drastic measures were being taken place to call patients like "do you really need this, or can you wait until June". You know things started to be more and more integrated into the telehealth way and Zoom was being used.-debriefing interview

\footnotetext{
Impact on quality of 3,4 care
}

One of the patients who has a lot of chronic illnesses..., he self-identified as someone whose not a phone person and is, notices himself that as engaged as much and getting distracted over the phone, and just is the kind of person that favors in person contact for a variety of reasons. And so, it really inhibited our work together and that he is less able to get into to a state of readiness to do therapeutic work because he's just distracted and then generally seeming feeling a lot more hopeless. -debriefing interview

\section{Use of PPE}

Continued 
Table 1 Continued

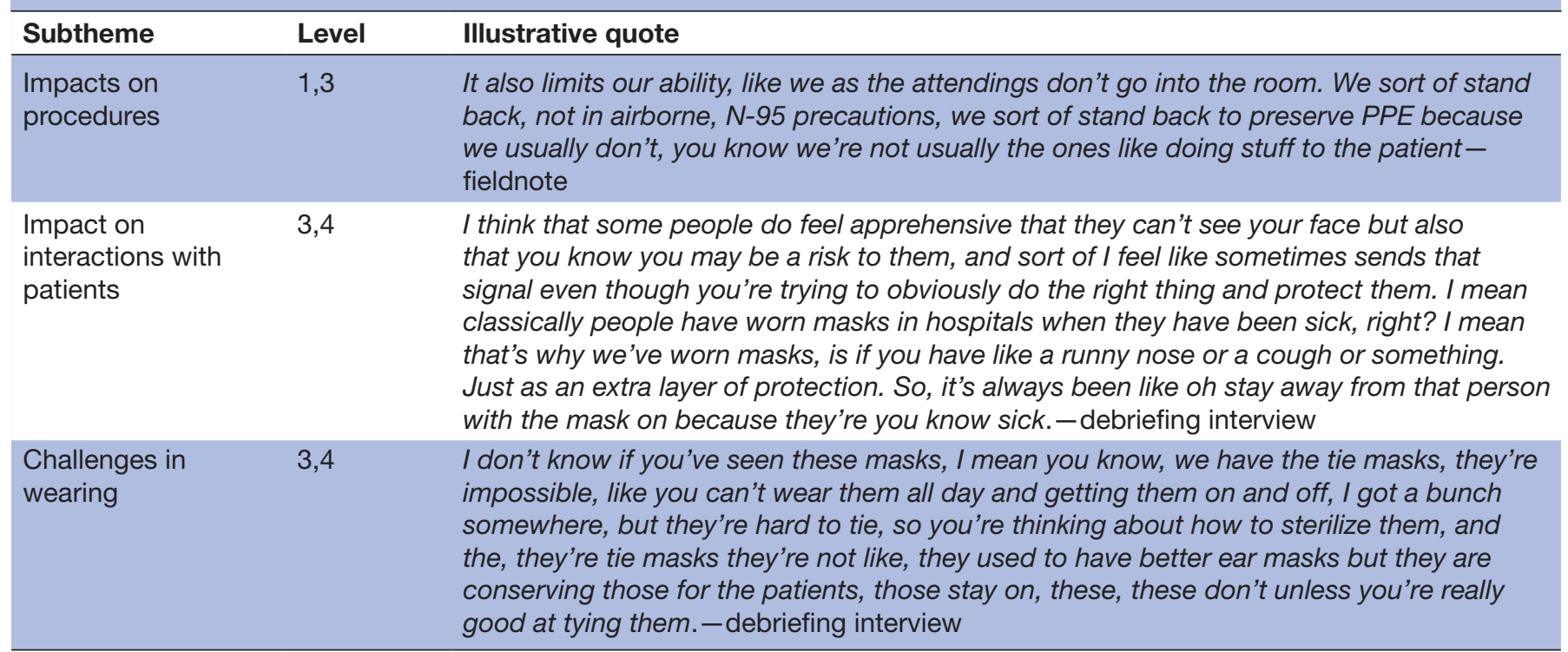

PPE, personal protective equipment.

as morning briefings and grand rounds with residents were either suspended or conducted remotely. Conferences with colleagues concerning patient clinical status and treatment were altered by requirements for physical separation (eg, limiting the number of providers in a patient's room, communicating remotely).

Perhaps the greatest impact of social distancing guidelines noted by POs was the restrictions on the presence of family members. This was especially problematic because the restrictions deprived patients of essential sources of social and emotional support, making it difficult for providers to communicate with family members and for family members to be updated on patient status and led to some patients dying alone without family members being present.

In some settings like behavioural health and outpatient psychiatry, there was a greater use of telehealth services. For the most part, these services were provided over the telephone or on the Zoom platform. Because of social distancing, some behavioural health consultations were performed without use of standard assessment protocols (ie, administration of questionnaires to evaluate mental health status). Moreover, some patients expressed reluctance or unwillingness to obtain treatment by telephone, making service delivery problematic. This reluctance led to concerns that such patients were not receiving optimal and necessary services.

\section{Use of PPE}

There are several facets of PPE use that were mentioned by providers, including policies that were designed to preserve the supply of PPEs in units like the operating rooms, challenges involved in wearing PPEs, including the time involved in 'donning and doffing' that created delays in performing procedures and the perceptual separation from patients created by the PPEs. Providers were required to undergo training in the use of PPEs and were monitored for proper use in the workplace. Some providers commented on the potential risk of infection created by improper use and the unwillingness of other providers to use PPEs in some units prior to the implementation of new guidelines mandating their use that replaced old guidelines that merely recommended their use.

\section{Theme 2: impacts on providers}

The second major theme is related to the impact of the pandemic in general and its impact on service delivery in particular to the providers themselves. This theme was segmented into three distinct subthemes (table 2): (1) risk of infection, (2) negative impacts and (3) provider coping strategies and resources.

\section{Risk of infection}

The first subtheme was provider assessments of the risk of infection to themselves and to family members. Unlike other healthcare systems where providers have died from COVID-19, there have been no known reported provider deaths in this healthcare system, even though it is widely recognised that some providers have tested positive for COVID-19. Nevertheless, although POs did report instances of a lack of concern by themselves or by others, sometimes reflected in the absence of masks worn in workspaces prior to the establishment of a policymaking their use mandatory, they also cited numerous instances of concern about getting infected. These concerns extended to the risk of infecting family members. The risk of infection was associated with factors such as the provider's age, occupation (eg, anaesthesiologists) and work setting (eg, operating room, Intensive Care Unit). 
Table 2 Impacts of COVID-19 pandemic on healthcare providers

$\begin{array}{lll}\text { Subtheme } & \text { Level } & \text { Illustrative quote } \\ \text { Risk of infection } & 1,3,4 & \begin{array}{l}\text {...the kind of thing that would really be unexpected and really upsetting is having } \\ \text { evaluated a patient, for instance, this week who was negative and then they [tested } \\ \text { positive), and for all of us to hear about that and then have to worry about that. } \\ \text { debriefing interview }\end{array}\end{array}$
debriefing interview

\section{Negative impacts}

Anxiety $1,3,4$

$1,3,4$

Depression $\quad 3,4$

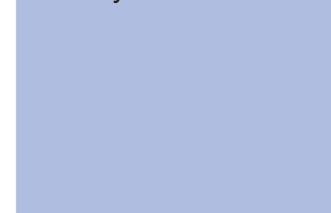

I mean there's a fair bit of anxiety, for sure. I think with regards to, you know exposure, family, sort of uncertainty. And just like trying to do the best you can in a different sort of world, if you want to call it that, with the COVID-19 sort of being the primary thing that comes up every step of the way. Like sometimes you're standing there and you're like oh my God this patient is bleeding to death, can we stop talking about the COVID-19? You know but it's something that we're just having, having to talk about. I think, I think that the anxiety part.- debriefing interview

It's been sad, just the effect that this has had on these 2 patients in particular. One
because I feel like that for months and months and months, we've been working together to get out more and to spend more time doing things, but, you know, give them a sense of purpose or satisfaction. It almost hurts them that much more, you know they've been working towards it, both of them had achieved the task of getting out more, so just as they were starting to get it together and like "oh this like really does work and this is really helping" and seeing some improvement and symptoms, and then it being taken away from them is pretty earth shattering. - debriefing interview

\begin{tabular}{ll} 
Stress & 4 \\
\hline Guilt & 4
\end{tabular}

There are providers that are stressed. I mean, it's the COVID-19 stress, it's the daycare stress, unemployment stress, kids not getting jobs. It's a whole morass, as you probably already know. things that are happening to people.-debriefing interview

Yeah, and I think people feel conflicted that you get to go to work and see your friends and so you get to have those at work and you get to have a conversation with adult friends in person and a lot of people don't get to do that anymore. And that sounds fun... I think there's also this is little bit of guilt in I know I told you that [the hospital] is not seeing this deluge of patients and you know, the community, the restaurants are giving out free lunch and local celebrities... have dropped off some food or some free thing to healthcare workers... and you're sort of like well actually we aren't seeing that many patients right now with COVID-19.--debriefing interview

\begin{tabular}{|c|c|c|}
\hline Ethical conflicts & 3,4 & $\begin{array}{l}\text { I think one of the early discussions we had... we have a program here where we use } \\
\text { ECMO for respiratory failure. And one of the early discussions we had here with not } \\
\text { just the hospital..., but also with other ECMO centers throughout the Pacific Northwest } \\
\text { was what are we going to do in the anticipation of this surge of patients? Does it } \\
\text { make sense to utilize a very high resource, you know procedure, for a very, very small } \\
\text { number of patients, where a lot of PPE is going to be used and a lot of dedication, a } \\
\text { lot of dedicated staff. And at that time, we kind of made the decision that we, that we } \\
\text { wouldn't...that did not make sense. That we wouldn't offer that service. As it started to } \\
\text { unfold, that, you know the surge that we were anticipating didn't develop quite in the } \\
\text { way that we thought it would or we feared that it would, we then kind of, as a group, } \\
\text { reinstituted the procedure and recognizing that, well it seems like we do have the } \\
\text { capacity both in terms of staff and space and with PPE and equipment to provide that } \\
\text { service.- debriefing interview }\end{array}$ \\
\hline Social tension & 4 & $\begin{array}{l}\text { My colleague that's been here for } 15 \text { years, she's great. At the end [of our shift] as we } \\
\text { were saying goodbye to her, she asks me to tell her everything you've learned [from } \\
\text { this study). She's pushing me; she said "okay [name removed), so why do you get to } \\
\text { do research? That's a pretty privileged thing to do and then why don't you come here } \\
\text { [to treat patients), I'm doing this yes you know, and you know it's also like we need } \\
\text { people."-debriefing interview }\end{array}$ \\
\hline \multicolumn{2}{|c|}{ Procedural innovations 3,4} & $\begin{array}{l}\text { We want to make sure that our outpatients clinic and providers are safe and patients } \\
\text { with COVID-19 go to outpatient units and so it's an important workaround but for } \\
\text { patients that will have trouble with Telemedicine and Telehealth, it does feel like the } \\
\text { emergency department is now not only a safety net but it's sort of the end of the road } \\
\text { for a lot of people-debriefing interview }\end{array}$ \\
\hline
\end{tabular}

Continued 
Table 2 Continued

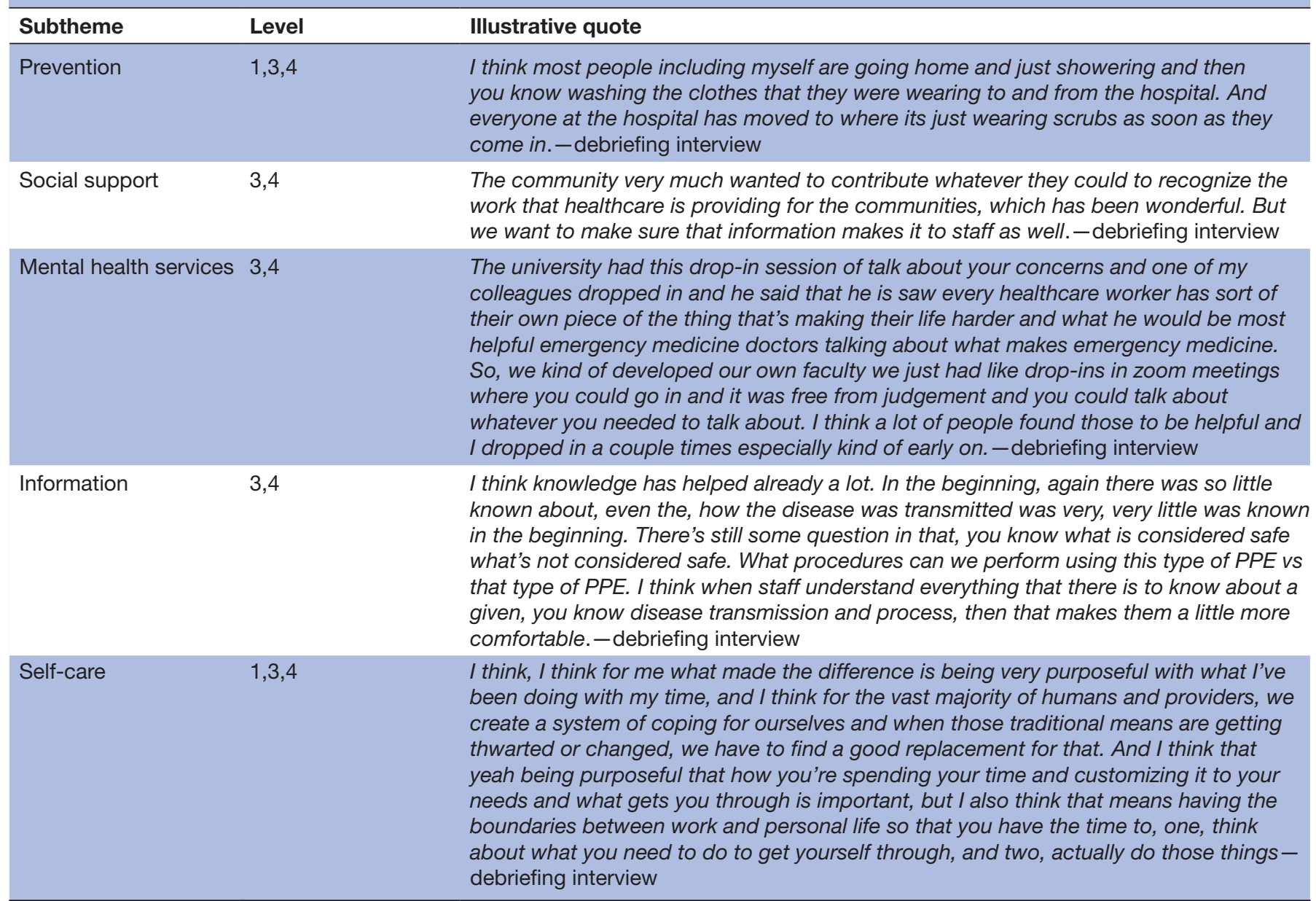

Negative impacts

Negative impacts of the pandemic on hospital staff, included anxiety related to the fear of infection to self and family members; feelings of sadness and depression related to separation of family members from dying patients and not being able to deliver necessary care, the experience of ethical tensions related to the perceived risk of coming to work sick and infecting others, engaging in other forms of risk behaviour like violating stay at home orders and the concern that some forms of care are currently being or will likely be rationed; guilt over having the opportunity to interact with colleagues when others must stay at home; interactions with colleagues that highlight undercurrents of social tension related to professional disciplinary differences (eg, research vs clinical care) or failure to adhere to guidelines regarding distancing and stress related to other aspects of the pandemic, including financial stability, impacts on loved ones and isolation and confinement at place of residence.

\section{Provider coping strategies and resources}

A third subtheme reflected different strategies and techniques employed by providers to cope with changes in service delivery and their impacts on both quality of care and on provider mental health. POs noted several instances of innovation in performing procedures while adhering to guidelines intended to protect both providers and patients from infection. These included adapting procedures for performing psychiatric evaluations for patients and development of workarounds to ensure service delivery.

A second important form of coping revolved around efforts to engage in behaviours and practices intended to reduce the risk of infection to self and others. These included behaviours at the workplace (use of homemade gels to clean hands or commercially available disinfectants to deep clean workspaces, not wearing street clothes or jewellery), outside of work (changing clothes before going shopping, practicing social distancing) and at home (changing clothes before going indoors, showering and physical separation, including staying in hotel rooms or air B\&Bs).

Social support was another significant coping resource reported by the POs. This included support provided by family members, some of whom were themselves healthcare providers and support from colleagues at work such as assistance in donning PPE, acquiring PPE and adjusting schedules to cover for colleagues at risk for infection and illness. It also included support from the community, 
manifested in deliveries of food and public expressions of gratitude.

A fourth important coping resource was the availability of mental health services. The healthcare system provided counselling services to providers and staff. These included drop-in sessions for all hospital employees with mental health service providers and drop-in sessions developed by individual units or departments within the system. Both types of sessions occurred over Zoom. Although the services provided were acknowledged to be helpful by those providers and staff who used them, there was also a sense that they were not widely used.

A fifth important resource was information. With experience and information provided by the system and preliminary research by others, the level of uncertainty associated with the pandemic, including risk of infection, duration of the pandemic and best practices for treatment, appeared to be diminishing, if only by degrees.

Finally, there were numerous reports of attempts at selfcare. These included a focus on healthy eating habits, adopting alternative forms of physical exercise, engaging in mindfulness and reflexivity and spending more time outdoors.

\section{Theme 3: impact on patients}

The third theme was the impact of the pandemic on the patients seen in the acute care setting. This theme included four subthemes (table 3): (1) patient access to care, (2) patient fears of getting infected at the hospital, (3) changes in presenting problems and (4) disparities in patient risk for COVID-19 and healthcare access.

\section{Patient access to care}

One of the biggest challenges faced by patients has been in getting access to care. The ED saw more patients who had appointments for non-essential care in other departments cancelled due to office closures. POs also noted changes in patient-provider interactions resulting from social distancing and PPE use and the suspension of non-essential procedures.

\section{Fear of getting infected at the hospital}

Patients expressed concerns about becoming infected while getting treated at the hospital and infecting family members in turn. Other patients have delayed getting medications refilled at the hospital to reduce the risk of infection.

Table 3 Impacts of COVID-19 pandemic on patients

\begin{tabular}{|c|c|c|}
\hline Subtheme & Level & Illustrative quote \\
\hline Access to care & $2,3,4$ & $\begin{array}{l}\text { Also, transitions for people seeking treatment have been difficult. Our detox center for } \\
\text { alcohol detox treatment now requires negative COVID-19 testing. Our outpatient based } \\
\text { opioid treatment program partner now only utilizes phone appointments. Many community } \\
\text { mental health programs are no longer accepting walk-ins. I'm hopeful this will change, } \\
\text { but service access for patients with SUD [substance use disorder] is really difficult right } \\
\text { now.- debriefing interview }\end{array}$ \\
\hline
\end{tabular}

\begin{tabular}{|c|c|c|}
\hline Presenting problems & $2,3,4$ & $\begin{array}{l}\text { We have not been as busy from a trauma perspective, although the last couple weeks have } \\
\text { been picking up as people, I think, are getting a little more antsy with the social distancing } \\
\text { and things. We've certainly seen a lot, like a lot more, or it seems like more at least of the } \\
\text { self-harm and non-accidental type of traumas, which has been challenge in and of itself. } \\
\text { And then on the general surgery side it seems like people with like normal problems like } \\
\text { appendicitis and you know infected gallbladders are coming in later than the otherwise } \\
\text { would I think out of concern for, you know, being in the hospital if they don't need to be } \\
\text { which is a valid concern.--debriefing interview }\end{array}$ \\
\hline
\end{tabular}




\section{Changes in presenting problems}

Some of the POs also noted more patients with mental and behavioural health issues that have been exacerbated by the threat of infection, collapse of the economy and the challenges in obtaining medication and non-essential clinical services. Delays in seeking or receiving services due to the pandemic were also perceived to result in patients presenting with more severe symptoms or clinical conditions when they are finally seen.

\section{Disparities in risk for infection}

Finally, the pandemic has illustrated the health disparities that have long been associated with the risk of illness and the accessibility of healthcare. Providers reported several instances of patients from disadvantaged backgrounds, including older adults, homeless, non-English-speaking immigrants, the poor and the disabled, who are overrepresented in acute care safety net settings under normal circumstances, but who also test positive for the novel coronavirus or are a COVID-19 person under investigation and who reside in households where the risk of transmission of the virus is high.

\section{Theme 4: overall impact on quality of care}

Despite concerns expressed by staff over the potential effects of delays in testing for COVID-19 and the challenges associated with social distancing and PPE use, the overall quality of care delivered to patients does not appear to have been significantly affected. This is attributed by providers and staff to four factors (table 4). First, the April 2020 surge was less than anticipated. After the initial outbreak of cases, the pandemic had more of an impact on assessment of cases that were coming in than on the number of patients actually treated. Workload did increase in many instances due to the imposition of new procedures related to PPE, distancing and coverage for personnel at risk for infection, but there was no sense that people were working longer hours, for instance. Second, the system was viewed by its employees as having been prepared for the pandemic from an operations perspective. With the initial outbreak at an assisted care nursing facility in a suburban community, a regional incidence response plan and hospital guidelines for patient screening, social distancing and PPE use were implemented. Some of those guidelines changed over time as the anticipated surge failed to materialise and as experience dictated necessary improvements to reduce delays and maintain standards for service delivery. Third, while some supplies such as N95 masks were in short supply and procedures for screening ED patients for COVID-19 were based on the perceived need to limit provider use of PPE to patients who tested positive or were at significant risk for infection, supplies viewed as essential for responding to the pandemic, including PPE and ventilators, were available and adequate to the current demand. Finally, despite the negative impacts on providers listed earlier, morale among hospital staff was high. Providers and staff appeared to be managing with the resources available to them that enable them to provide the best care possible, seek emotional support, engage in self-care and exercise preventive measures designed to reduce the risk of infection.

\section{DISCUSSION}

This study identified four different kinds of impacts of the COVID-19 pandemic on delivery of clinical services in a level 1 trauma centre during a surge of cases that occurred the month of April 2020: procedural, provider,

Table 4 Overall impacts of COVID-19 pandemic on service delivery

\begin{tabular}{|c|c|c|}
\hline Subtheme & Level & Illustrative quote \\
\hline $\begin{array}{l}\text { Fewer cases than } \\
\text { expected }\end{array}$ & 1,2 & $\begin{array}{l}\text { Yeah, so we, you know we did prep for a much larger surge based on the initial } \\
\text { predictions for Washington than we ended up having. I think as a result of pretty } \\
\text { aggressive social distancing and stay at home orders, which if you look at them, } \\
\text { the series of prediction sort of the surge got less and less. - debriefing interview }\end{array}$ \\
\hline System was prepared & 2,3 & $\begin{array}{l}\text { At Harborview though, you know, we received patients from that event. It was not, } \\
\text { it did not overwhelm us. We then, you know that sort of triggered the overall, sort } \\
\text { of regional, you know, incident response structure that is in place today. And as } \\
\text { we started to prepare for the surge, we were able to very easily keep up with the } \\
\text { inflow of patients. And so, at this point the workload... you know people are still } \\
\text { very much able to get their time off. The workload is, I mean there's work to be } \\
\text { done but it's not overwhelming. And so, I think from that standpoint, we haven't } \\
\text { seen the fatigue, the long hours, the multiple days, that you might see where, you } \\
\text { know, kind of the picture that's being described in the, in New York right now.- } \\
\text { debriefing interview }\end{array}$ \\
\hline
\end{tabular}

$\begin{array}{lll}\begin{array}{l}\text { Supplies were adequate } \\ 2,3\end{array} & 3,4 & \begin{array}{l}\text { So, so the provider saw the } 20 \text { patients on the unit. And got ample goggles, masks } \\ \text { and gloves on the unit from the nursing staff. - jotting }\end{array} \\ \text { High staff morale } & \begin{array}{l}\text { So, it's definitely, it's definitely something on people's minds. But does it affect } \\ \text { the day-to-day performance? I have not seen that. People are absolutely willing to } \\ \text { step in and do the work. - debriefing interview }\end{array}\end{array}$


patient and overall. Each impact highlighted two or more levels of a socioecological model of service delivery: the outermost or environmental service setting framed by the novel coronavirus and its global spread, the external or macroservice setting framed by the supply and demand for care; the internal or mezzo service setting framed by guidelines and policies put in place to ensure the safety and health of both patients and providers and the micro service setting framed by individual patient and provider behaviour. Despite significant changes in procedures that included COVID-19 screening of all admitted patients, social distancing and use of PPE, as well as changes in patient characteristics and provider behaviour, the overall impact of the pandemic on the quality of service delivery, as described by frontline providers, appears to have been minimal. This is attributed to having a smaller surge than expected, a quick response by the healthcare system to anticipated demands for service delivery and protection of patients and providers, available supplies and high provider morale.

Consistent with studies of earlier infectious disease pandemics $^{13-23}$ and recent reports published during the early phases of the COVID-19 pandemic in China, ${ }^{40}$ Italy ${ }^{41}$ and the USA, ${ }^{42}$ reports of anxiety and fear of infection among trauma centre providers and staff were widespread. Providers also reported instances of stress related to other aspects of the pandemic, including financial stability, impacts on loved ones and isolation and confinement, which have also been found in studies of other pandemics. ${ }^{15} 16$ However, there were also reports of depressed mood related to separation of family members from sick and dying patients and not being able to deliver necessary care, the experience of ethical tensions related to the perceived risk of coming to work sick and infecting others, engaging in other forms of risk behaviour like violating stay at home orders and the concern that some forms of care were currently being or likely to be rationed; guilt over having the opportunity to interact with colleagues when others must stay at home; and interactions with colleagues that highlight undercurrents of social tension related to professional disciplinary differences or failure to adhere to guidelines regarding distancing. These impacts have not been reported in previous studies of the psychological impacts of other infectious disease pandemics on healthcare providers. ${ }^{13-22}$

It is also quite likely that levels of anxiety and fear of infection were much less than has been reported in other healthcare systems because the surge was much less than anticipated and because there were no reports of providers and staff becoming severely ill or dying despite a positive test. ${ }^{31}$ Earlier studies of ED personnel and infectious disease pandemics have also noted lower than expected prevalence of mental health problems, which have been attributed to the greater resilience of individuals who choose this type of work. ${ }^{21}$ We also identified several strategies used by providers and staff to cope with the pandemic and its organisational and individual impacts. Adaptive coping has been associated with reduced risk of psychiatric morbidity and has been reported in studies of other respiratory disease outbreaks. ${ }^{12} 161721$

The study occurred in a healthcare setting that was one of the firsts to be impacted by the pandemic. However, the impacts associated with the pandemic in this setting have not been as severe as has been the case elsewhere, especially in New York city, limiting the generalisability of our findings. Furthermore, our findings are limited by the relative short duration of participation observation (1-4 weeks) in a single setting (trauma/emergency medicine) and the constraints of engaging in participant observation while also performing intensive clinical tasks under conditions of social distancing and use of PPE. In contrast to studies of previous infectious disease pandemics, ${ }^{131417182021}$ no standardised measures were used to assess mental health status. Our assessment of impacts on the quality of service delivery was based entirely on self-report or observational data and not on objective measures of quality of care.

Despite these limitations, this study was one of the first to be conducted in the USA that examined the impact of a still-unfolding infectious disease pandemic in a healthcare setting representing the first point of entry for patients with COVID-19 positive. Although previous studies of healthcare responses to infectious disease pandemics have also noted changes in procedures, ${ }^{13} 1518$ this is the first study to our knowledge to examine the impact of these changes on service delivery. The study used a standardised protocol for conducting ethnographic research that enabled us to collect and analyse data in a short period of time with minimal impact on patients or providers under conditions of social distancing and PPE use. The RAPICE approach also has potential for assessing these impacts longitudinally and providing formative evaluations of policies and procedures designed to mitigate them.

\section{CONCLUSIONS}

Although this study was conducted within one setting in one healthcare system in one community, the findings offer some important lessons for healthcare systems that have yet to be impacted as well as systems that have been more severely impacted. Each of the levels in our socioecological model were found to impact the delivery of services to patients in the time of COVID-19 and variations at each of these levels account for variations in that delivery of care globally.

\section{Author affiliations}

${ }^{1}$ Suzanne Dworak-Peck School of Social Work, University of Southern California, Los Angeles, California, USA

${ }^{2}$ Emergency Medicine, University of Washington School of Medicine, Seattle, Washington, USA

${ }^{3}$ Surgery, University of Washington School of Medicine, Seattle, Washington, USA ${ }^{4}$ Psychiatry \& Behavioral Sciences, University of Washington School of Medicine, Seattle, Washington, USA

${ }^{5}$ Division of Trauma, Burn and Critical Care Surgery, Harborview Medical Center, Seattle, Washington, USA 
Contributors LAP and DZ conceived and designed the study and the analysis plan. LW, DN, AE, MT and DFZ collected the data and participated in data analysis, along with LAP. KM provided study project management. All authors contributed intellectual content during the drafting and revision of the work and approved the final version.

Funding This study was supported in part by the Patient-CenteredCentred Outcomes Research Institute (PCORI) Award (IHS-2017C1-6151). This research was also supported within the National Institutes of Health (NIH) Health Care Systems Research Collaboratory by cooperative agreement (4UH3MH106338-02) from the National Institute of Mental Health. Support was also provided by the NIH Common Fund through cooperative agreement (U24AT009676) from the Office of Strategic Coordination within the Office of the NIH Director.

Competing interests All authors had financial support from the PatientCenteredCentred Outcomes Research Institute and National Institutes of Health for the submitted work.

\section{Patient consent for publication Not required}

Ethics approval All study procedures were approved by the IRBs of the University of Washington and University of Southern California (UP-20-00298) prior to the initiation of the investigation.

Provenance and peer review Not commissioned; externally peer reviewed.

Data availability statement Data used in this study are available from the corresponding author upon reasonable request. All personal identifiers found in the data will be removed prior to sharing.

Supplemental material This content has been supplied by the author(s). It has not been vetted by BMJ Publishing Group Limited (BMJ) and may not have been peer-reviewed. Any opinions or recommendations discussed are solely those of the author(s) and are not endorsed by BMJ. BMJ disclaims all liability and responsibility arising from any reliance placed on the content. Where the content includes any translated material, BMJ does not warrant the accuracy and reliability of the translations (including but not limited to local regulations, clinical guidelines, terminology, drug names and drug dosages), and is not responsible for any error and/or omissions arising from translation and adaptation or otherwise.

Open access This is an open access article distributed in accordance with the Creative Commons Attribution Non Commercial (CC BY-NC 4.0) license, which permits others to distribute, remix, adapt, build upon this work non-commercially, and license their derivative works on different terms, provided the original work is properly cited, appropriate credit is given, any changes made indicated, and the use is non-commercial. See: http://creativecommons.org/licenses/by-nc/4.0/.

\section{ORCID iD}

Douglas F Zatzick http://orcid.org/0000-0001-7339-1020

\section{REFERENCES}

1 Cucinotta D, Vanelli M. Who Declares COVID-19 a pandemic. Acta Biomed 2020;91:157-60.

2 Taubenberger JK, Kash JC, Morens DM. The 1918 influenza pandemic: 100 years of questions answered and unanswered. Sci Transl Med 2019;11:eaau5485.

3 The Johns Hopkins University and School of Medicine. Coronavirus center. COVID-19 dashboard by the center for systems science and engineering (CSSE) at Johns Hopkins University. Available: https:// coronavirus.jhu.edu/map.html [Accessed 29 May 2020].

4 Bureau of Labor Statistics. Unemployment rate rises to record high 14.7 percent in April, 2020. Available: https://www.bls.gov/opub/ted/ 2020/unemployment-rate-rises-to-record-high-14-point-7-percentin-april-2020.htm?view_full [Accessed 15 May 2020].

5 California coronavirus (COVID-19) response. stay home except for essential needs. Available: https://covid19.ca.gov/stay-homeexcept-for-essential-needs/\#top [Accessed 4 May 2020].

6 Morganstein JC, Fullerton CS, Ursano RJ, et al. Pandemics: health care emergencies. In: Raphael B, Fullerton CS, Weisaeth L, et al, eds. Textbook of disaster psychiatry. 2nd ed. New York: Cambridge University Press, 2017: 270-84.

7 Rubinson L, Mutter R, Viboud C, et al. Impact of the fall 2009 influenza $\mathrm{A}(\mathrm{H} 1 \mathrm{~N} 1)$ pdm09 pandemic on US hospitals. Med Care 2013;51:259-65.

8 Schanzer DL, Schwartz B. Impact of seasonal and pandemic influenza on emergency department visits, 2003-2010, Ontario, Canada. Acad Emerg Med 2013;20:388-97.
9 Panagioti M, Geraghty K, Johnson J, et al. Association between physician burnout and patient safety, professionalism, and patient satisfaction: a systematic review and meta-analysis. JAMA Intern Med 2018;178:1317-30.

10 Tawfik DS, Scheid A, Profit J, et al. Evidence relating health care provider burnout and quality of care: a systematic review and metaanalysis. Ann Intern Med 2019;171:555-67.

11 Benedek DM, Fullerton C, Ursano RJ. First responders: mental health consequences of natural and Human-Made disasters for public health and public safety workers. Annu Rev Public Health 2007;28:55-68.

12 Naushad VA, Bierens JJ, Nishan KP, et al. A systematic review of the impact of disaster on the mental health of medical responders. Prehosp Disaster Med 2019;34:632-43.

13 Nickell LA, Crighton EJ, Tracy CS, et al. Psychosocial effects of SARS on hospital staff: survey of a large tertiary care institution. CMAJ 2004;170:793-8.

14 Chua SE, Cheung V, Cheung C, et al. Psychological effects of the SARS outbreak in Hong Kong on high-risk health care workers. Can $J$ Psychiatry 2004;49:391-3.

15 Bai Y, Lin C-C, Lin C-Y, et al. Survey of stress reactions among health care workers involved with the SARS outbreak. Psychiatr Serv 2004;55:1055-7.

16 Wong TW, Yau JKY, Chan CLW, et al. The psychological impact of severe acute respiratory syndrome outbreak on healthcare workers in emergency departments and how they cope. Eur J Emerg Med 2005;12:13-18.

17 Lin C-Y, Peng Y-C, Wu Y-H, et al. The psychological effect of severe acute respiratory syndrome on emergency department staff. Emerg Med J 2007;24:12-17.

18 Goulia P, Mantas C, Dimitroula D, et al. General Hospital staff worries, perceived sufficiency of information and associated psychological distress during the $\mathrm{A} / \mathrm{H} 1 \mathrm{~N} 1$ influenza pandemic. $B M C$ Infect Dis 2010;10:322.

19 Al Ghobain M, Aldrees T, Alenezi A, et al. Perception and attitude of emergency room resident physicians toward middle East respiratory syndrome outbreak. Emerg Med Int 2017;2017:1-4.

20 Lancee WJ, Maunder RG, Goldbloom DS, et al. Prevalence of psychiatric disorders among Toronto Hospital workers one to two years after the SARS outbreak. Psychiatr Serv 2008;59:91-5.

21 Maunder RG, Lancee WJ, Balderson KE, et al. LongTerm psychological and occupational effects of providing Hospital healthcare during SARS outbreak. Emerg Infect Dis 2006;12:1924-32.

22 Lai J, Ma S, Wang Y, et al. Factors associated with mental health outcomes among health care workers exposed to coronavirus disease 2019. JAMA Netw Open 2020;3:e203976.

23 Wilder-Smith A, Chiew CJ, Lee VJ. Can we contain the COVID-19 outbreak with the same measures as for SARS? Lancet Infect Dis 2020;20:e102-7.

24 Matrajt L, Leung T. Evaluating the effectiveness of social distancing interventions to delay or flatten the epidemic curve of coronavirus disease. Emerg Infect Dis 2020;26:1740-8.

25 Mahler J. Epicenter: inside the underfunded, overwhelmed public hospitals that are trying to save New York. The New York Times Magazine 2020:24-51.

26 Kim CS, Lynch JB, Cohen S, et al. One academic health system's early (and ongoing) experience responding to COVID-19: recommendations from the initial epicenter of the pandemic in the United States. Acad Med 2020;95:1146-8.

27 McLeroy KR, Bibeau D, Steckler A, et al. An ecological perspective on health promotion programs. Health Educ Q 1988;15:351-77.

28 Kumar S, Quinn SC, Kim KH, et al. The social ecological model as a framework for determinants of $2009 \mathrm{H} 1 \mathrm{~N} 1$ influenza vaccine uptake in the United States. Health Educ Behav 2012;39:229-43.

29 Moore M, Cristofalo M, Dotolo D, et al. When high pressure, system constraints, and a social justice mission collide: a socio-structural analysis of emergency department social work services. Soc Sci Med 2017;178:104-14.

30 Scheuer $\mathrm{H}$, Engstrom A, Thomas $\mathrm{P}$, et al. A comparative effectiveness trial of an information technology enhanced peer-integrated collaborative care intervention versus enhanced usual care for us trauma care systems: clinical study protocol. Contemp Clin Trials 2020;91:105970.

31 Palinkas LA, Zatzick D. Rapid assessment procedure informed clinical ethnography (RAPICE) in pragmatic clinical trials of mental health services implementation: methods and applied case study. Adm Policy Ment Health 2019;46:255-70.

32 Moloney K, Scheuer H, Engstrom A, et al. Experiences and insights from the early us COVID-19 epicenter: a rapid assessment procedure informed clinical ethnography case series. Psychiatry 2020;83:115-27. 
33 Palinkas LA, Prussing E, Reznik VM, et al. The San Diego East County school shootings: a qualitative study of community-level post-traumatic stress. Prehosp Disaster Med 2004;19:113-21.

34 Zatzick D, Coq N, Frederic J, et al. Psychosocial support training for HIV health care providers in response to the Haitian earthquake. Consortium of. Seattle, WA: Universities for Global Health Annual Meeting, University of Washington, 2010.

35 HCPro. Case study: Harborview Medical Center's automated sepsis alert system. Nurse Leader Insider Sept 6, 2018. Available: https:// www.hcpro.com/NRS-331768-868/Case-Study-Harborview-MedicalCenters-Automated-Sepsis-Alert-System.html [Accessed 9 Jun 2020].

36 Padgett DK. Qualitative methods in social work research. 3rd edn. Los Angeles, CA: Sage, 2017.

37 Miller WL, Crabtree BF. Primary care research: a multimethod typology and qualitative road map. In: Crabtree BF, Miller WL, eds. Doing qualitative research. Newbury Park, CA: Sage, 1992: 3-30.

38 Saldana J. The coding manual for qualitative researchers. 3rd ed. Los Angeles: Sage, 2016.
39 Centers for Disease Control. Social distancing. National center for immunization and respiratory diseases (NCIRD), division of viral diseases. Available: https://www.cdc.gov/coronavirus/2019ncov/prevent-getting-sick/social-distancing.html\#: :text=Social\% 20distancing\%2C\%20also\%20called\%20\%E2\%80\%9Cphysical, both\%20indoor\%20and\%20outdoor\%20spaces [Accessed 26 Aug 2020].

40 Chen Q, Liang M, Li Y, et al. Mental health care for medical staff in China during the COVID-19 outbreak. Lancet Psychiatry 2020;7:e15-16.

41 Barello S, Palamenghi L, Graffigna G. Burnout and somatic symptoms among frontline healthcare professionals at the peak of the Italian COVID-19 pandemic. Psychiatry Res 2020;290:113129.

42 Shanafelt T, Ripp J, Trockel M. Understanding and addressing sources of anxiety among health care professionals during the COVID-19 pandemic. JAMA 2020;323:2133. 